\title{
Three Decades of Research in Human mobility: A Scientomet- ric Analysis
}

1 Ph.D. in Geography and Rural Planning, Faculty of Earth Sciences, Shahid Beheshti University, Tehran, Iran; negin.mirvahedi1@gmail.com

2 Ph.D. Candidate of Geography and Urban Planning, Faculty of Humanities, Tarbiat Modares University, Tehran, Iran; a.doorudinia@modares.ac.ir

* Correspondence: negin.mirvahedi1@gmail.com

\begin{abstract}
This study aims to review and analyze research in human mobility as one of the main topics of study in urban and regional planning; Which studies the movement of human beings, as individuals or groups, in space and time. The research method is descriptive and uses scientometric, bibliographic analysis methods and citation analysis. The statistical population of this study is a collection of 2247 articles published in this field based on a search in the Scopus citation database. VOSviewer software was used for analysis. Findings showed that the publication of articles in this field has gone up during the years under review, and the most scientific productions have been in 2021 and 2020, respectively. Based on the clustering of high-frequency words, the topics of "Covid19", "mobility," "mobile phone data," "big data," and "migration" were identified as thematic clusters in this field. The United States and China also had the most significant number of articles. Gonzalez is the most cited researcher and the center for spatial information science, university of Tokyo has the most significant number of articles (11 articles) among scientific institutes active in this field. In contrast, the most cited and influential articles in this field belong to the Fogarty international center, united states (1196 citations in 4 articles). A combination of terms such as "covid-19" and "big data" in the mapping of science reflects efforts of the academic community active in this field of study to expand applied and up-to-date studies in this field.
\end{abstract}

Keywords: Human Mobility, Mobility, Scientometrics, Citation Analysis, Text mining.

Citation: Mirvahedi , N.; Doorudinia , A., 2022, Three Decades of Research in Human mobility: A Scientometric Analysis. SUPTM 2022 conference proceedings sciforum052978 .

https://doi.org/10.31428/10317/10485

Publisher's Note: UPCT and Sciforum stays neutral with regard to jurisdictional claims in published maps and institutional affiliations.

Copyright: (c) 2022 by the authors. Submitted for possible open access publication under the terms and conditions of the Creative Commons Attribution (CC BY) license (https://creativecommons.org/license s/by/4.0/).

\section{ords: Human Mobility, Mobility, Scientometrics, Citation Analysis, Text mining.}

\section{Introduction}

Understanding individual human mobility is essential for many areas of study, from urban and regional planning, traffic, demographics, epidemics [1]. The breadth of studies in this field requires a general overview of the status of research, how different fields are related, knowledge of how these fields grow and develop over time. One way to achieve these goals is scientometrics. The scientometric analysis is a quantitative analysis used to extract patterns and samples in a subject area or discipline [2]. By examining the process of science production in this field, a picture of scientific maps can be provided. In the evaluation and measurement methods of scientific products, quantitative data related to the production, distribution, and use of scientific texts, science, and scientific research can be described and reviewed, and its characteristics can be determined. Given the importance of human mobility and since there is no comprehensive research in this field, the purpose of this study is to analyze the scientometrics of scientific products in the field of human mobility.

\section{Methodology}

In this section, we apply the scientometric mapping approach in our collection of articles concerning their specific role in the intellectual structures of human mobility studies. This analysis began with downloading the citation information for our collection of 
articles from the Scopus database and importing them into the specific tools listed above. This research is applied research and is done using a scientometric method, bibliographic analysis methods, and a citation network. Scientometrics is the knowledge of measuring science; Which includes all quantitative methods and models related to producing and disseminating knowledge and technology. The study's statistical population includes all scientific productions indexed (2247 titles) in the Scopus database from 1986-2021 about human mobility. A data collection tool is also a note-taking tool, and a data analysis tool is Excel Vosviewer software. First, the selected data is collected by searching for keywords related to the research topic by forming a database, and then maps, structures, and clusters will be created using the software. In the last stage, after analyzing the information obtained, their relationships are discussed and concluded.

\section{Results}

\subsection{Overview of the data set}

Overall, as shown in Figure 1(a), research in human mobility has grown significantly since 2007. Initially, this field of research was minimal, and from 1986 to 2006, only 41 documents were published during the first 20 years. However, more than $98 \%$ of the collected documents were published from 2007 to 2021. This issue means that the literature on human mobility has recently received increasing attention and is in its infancy. The collection of documents published in the thematic field of human mobility has been published in journals with different thematic categories. For example, we can refer to the number of journals such as Plos One, EPJ Data Science, Scientific Reports, Physica A Statistical Mechanics and Its Applications, Journal of The Royal Society Interface, Proceedings of The National Academy of Sciences of The United States of America, ISPRS International Journal of Geo-Information and International Journal of Geographical Information Science. These show that human mobility has become an interdisciplinary topic. Figure 1(b) shows the ten topics with the largest share in the collection of 2247 selected documents that computer science, engineering, social sciences, mathematics, environmental sciences, respectively, form the main currents of research in the field of human mobility.

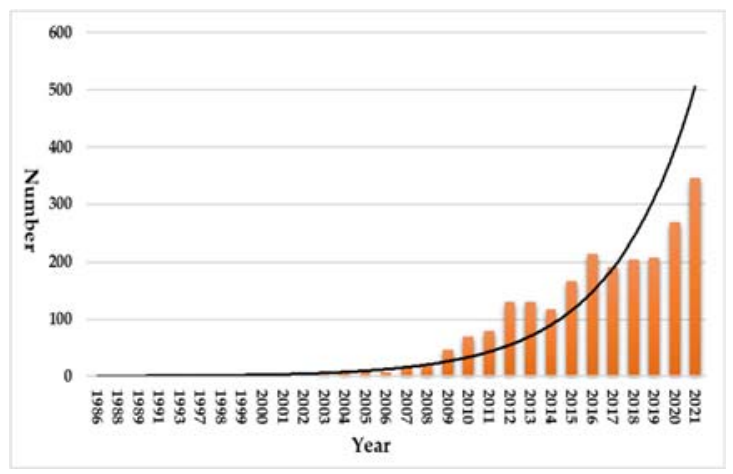

(a)

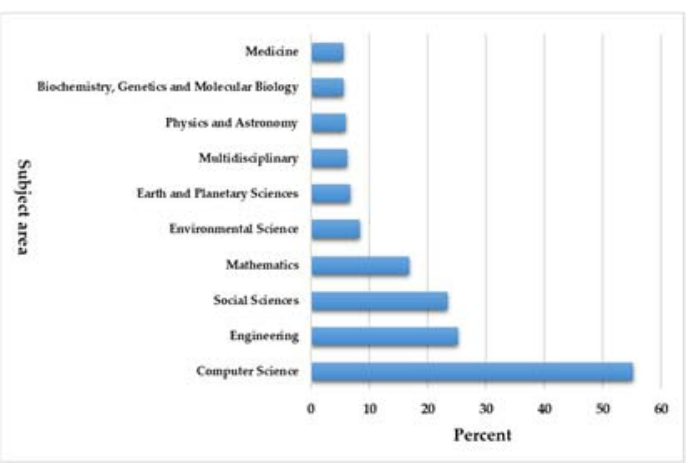

(b)

Figure 1. (a) Density Visualization of Co- Occurrence of keywords; (b) Ten subject areas with the largest share in the dataset.

Figure 2(a), shows a keyword map using content analysis created in VOS viewer software. Co-occurrence of keywords indicates the degree of connection between a set of documents [3]. In lexical analysis, it is assumed that the most frequent words have a more significant impact on a domain than the less frequent words. In addition, synoptic analysis allows us to identify emerging thematic clusters and develop clusters to predict the direction of future research [4]. As this image of keyword synchronization parsing shows, the primary expressions of our search in the literature, this subject area of human mobility, are at the center of the map. This keyword is surrounded by a set of signal words related to conceptualization (e.g., mobility, forecasting, behavioral research, location, migration, travel, mobility pattern, trajectories, population statistics) with new ideas (cellular 
telephones, covid-19, social networking (online), big data and data mining), Big data and data mining) or the new network are surrounded. These signal words reflect the main content of the new literature that is receiving widespread attention. This figure shows that human mobility has changed a lot with the advent of the information age and advances in the subject area. In addition, the distance between keywords indicates the rate at which they occur simultaneously in an article, which indicates the relationship between the words. In this regard, much attention has been paid to "Covid 19" among the signal words around. This issue highlights the importance of this area of research on the prevalence of the corona pandemic. Given the terms of the highest frequency signal, it is evident that "social networks" and "big data" have become the leading research fields in the study of human mobility.

\subsection{Mapping Intellectual Structures}

One of the things that makes a researcher influential in a field of expertise is how he or she interacts with other researchers. The ability to influence others in this way gives that researcher more influence. In other words, a researcher has a more substantial influence who can change their thoughts through social interactions with other researchers [5]. Co-authors are social networks that include authors and researchers of articles, each of which communicates with one or more other authors through references to their work in their articles, provided that they co-author one or more articles [6]. The premise behind this analysis is that when two things (for example, an author and a magazine) often appear together in an article, it can be said that they are somehow related [7]. In this regard, we visualized the co-authorship patterns of our collection of articles (Figure 2(b)) and presented the statistical results of the journal citation analysis (Table 1) as well as the author citation analysis (Table 2) by VOS viewer. Journal citation analysis can reflect the relationships between journal types and disciplines. Table 1 shows the most cited journals as citation sources for human mobility studies based on the internal citations of the selected collection of articles. The subject area and classification of journals are generally multidisciplinary, humanities, mathematics, computer science, social sciences, engineering, social sciences, medical sciences, and environmental sciences, and it shows that research in the field of human mobility has become more interdisciplinary. From 1986 to 2021, with the number of internal citations 1853, Nature magazine has the highest absolute frequency of internal citations. The journal Plus One and Science are the following significant sources of knowledge for this field of study.

Table 1. The top 5 most-cited journals

\begin{tabular}{ccc}
\hline $\begin{array}{c}\text { Frequency of } \\
\text { Citations }\end{array}$ & Journal & Subject Area and Category \\
\hline 1853 & Nature & Multidisciplinary \\
1542 & Plos One & Multidisciplinary \\
1389 & Science & Arts and Humanities - \\
& Proceedings Of The National Academy Of Sciences Of & Multidisciplinary \\
297 & The United States Of America & Multidisciplinary \\
282 & Physical Review E & Mathematics- Physics and \\
& & Astronomy \\
\hline
\end{tabular}

The joint writing map of the collection of articles in VOS viewer software consists of 4679 authors (Figure 2(b)). Nodes in this network represent authors, and the attribute of total link strength indicates the total power of a particular author's co-author links with other authors. In order to evaluate productivity and impact, Table 2 presents the ten authors with the most total citation power. Accordingly, Li, Liu, and Tatem are the most constructive authors in the dataset. 


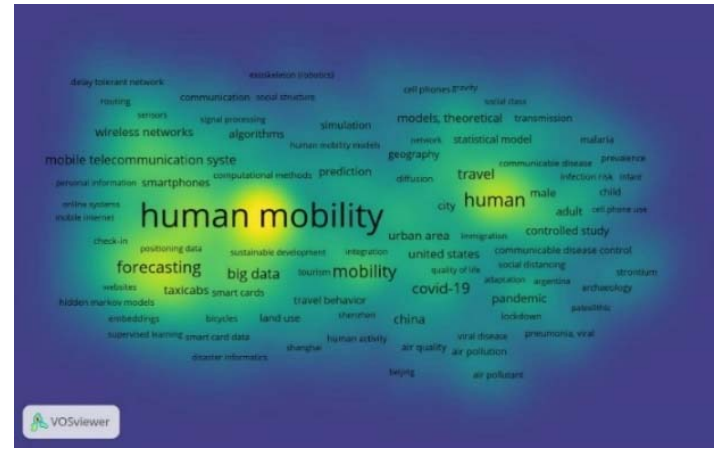

(a)

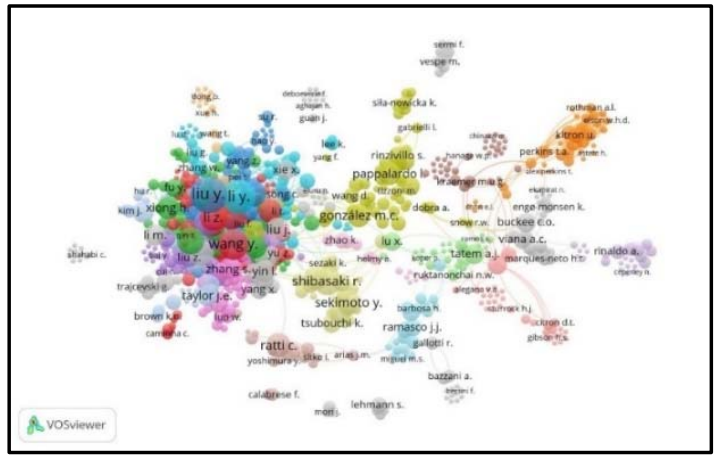

(b)

Figure 2. (a) Density Visualization of Co- Occurrence of keywords; (b) Co-authorship map of all authors from the set of 2247 papers.

Table 1. The top 10 most-cited journals

\begin{tabular}{cccccccccc}
\hline No & author & Documents & citations & Total link Strength & No & author & Documents & citations & Total link Strength \\
\hline 1 & li y. & 38 & 522 & 152 & 6 & kitron u. & 7 & 234 & 83 \\
2 & liu y. & 44 & 1501 & 148 & 7 & morrison a.c. & 7 & 234 & 83 \\
3 & tatem a.j. & 13 & 1518 & 105 & 8 & scott t.w. & 7 & 234 & 83 \\
4 & smith d.l. & 12 & 893 & 97 & 9 & zhang f. & 22 & 472 & 78 \\
5 & shibasaki r. & 26 & 690 & 90 & 10 & li z. & 24 & 196 & 77 \\
\hline
\end{tabular}

\section{Conclusion}

In this study, a scientometric study in human mobility studies was conducted. Accordingly, results show that in the last 15 years, the production of science in this field has been growing and has also become an interdisciplinary scientific subject. Entering the information age has been another factor in the evolution of studies in this field. In addition, the prevalence of the corona pandemic has quickly become one of the research interests of researchers active in this field. With the acceleration of the production of big data and especially the data produced by social networks in the world, studies related to this field will be much more extensive and will also undergo a growing trend. Therefore, more extensive studies will be needed to clarify paradigm shifts with scientometric methods.

Funding: This research received no external funding.

Conflicts of Interest: The authors declare no conflict of interest.

\section{References}

1. Jurdak R, Zhao K, Liu J, AbouJaoude M, Cameron M, Newth D. Understanding human mobility from Twitter. PloS one. 2015;10(7):e0131469. https://doi.org/https://doi.org/10.1371/journal.pone.0131469

2. Zhang X. A bibliometric analysis of Second Language Acquisition between 1997 and 2018. Studies in Second Language Acquisition. 2020;42(1):199-222. https://doi.org/https://doi.org/10.1017/S0272263119000573

3. Maditati DR, Munim ZH, Schramm H-J, Kummer S. A review of green supply chain management: From bibliometric analysis to a conceptual framework and future research directions. Resources, Conservation and Recycling. 2018; 139: 150-62. https://doi.org/https://doi.org/10.1016/j.resconrec.2018.08.004

4. Lee P-C, Su H-N. Investigating the structure of regional innovation system research through keyword co-occurrence and social network analysis. Innovation. 2010;12(1):26-40. https://doi.org/https://doi.org/10.5172/impp.12.1.26

5. Truex D, Cuellar M, Takeda H. Assessing scholarly influence: Using the Hirsch indices to reframe the discourse. Journal of the Association for Information Systems. 2009;10(7):1. https://doi.org/10.17705/1jais.00203

6. Khasseh AA, Soosaraei M, Fakhar M. Cluster Analysis and Mapping of Iranian Researchers in the Field of Parasitology: With an Emphasis on the Co-authoreship Indicators and H Index. Iranian Journal of Medical Microbiology. 2016;10(2):63-74.

7. Liu C, Gui Q. Mapping intellectual structures and dynamics of transport geography research: a scientometric overview from 1982 to 2014. Scientometrics. 2016;109(1):159-84. https://doi.org/https://doi.org/10.1007/s11192-016-2045-8 